Protocolo de análisis descriptivo de estrategias heurísticas aplicadas a la resolución de problemas Minotta Carlos (pp. 95-118)

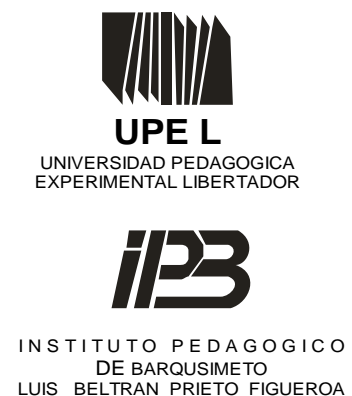

LUIS BELTRAN PRIETO FIGUEROA

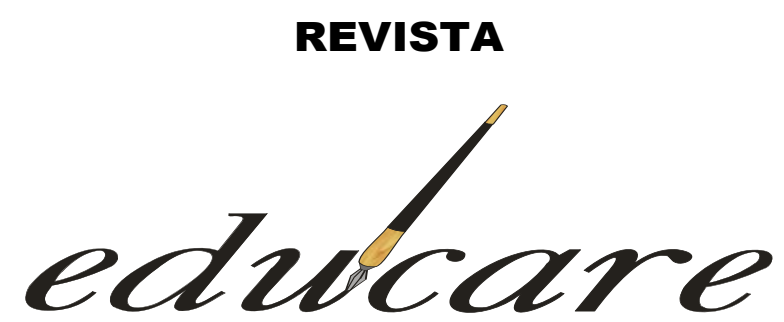

Órgano Divulgativo de la Subdirección de Investigación y Postgrado del Instituto Pedagógico de Barquisimeto "Luis Beltrán Prieto Figueroa”

BARQUISIMETO - EDO. LARA - VENEZUELA

NUEVA ETAPA

FORMATO ELECTRÒNICO

DEPOSITO LEGAL: PPI2O1002LA3674 VOLUMEN 19 № 1

ISSN: 2244-7296

ENERO - ABRIL 2015

\title{
PROTOCOLO DE ANÁLISIS DESCRIPTIVO DE ESTRATEGIAS HEURÍSTICAS APLICADAS A LA RESOLUCIÓN DE PROBLEMAS
}

\section{DESCRIPTIVE ANALYSIS PROTOCOL OF HEURISTIC STRATEGIES APPLIED TO PROBLEM SOLVING}

\section{MinOtTA CARLOS*}

* Universidad de ANTIOQuia, MEdelLín (Colombia) 


\section{PROTOCOLO DE ANÁLISIS DESCRIPTIVO DE ESTRATEGIAS HEURÍSTICAS APLICADAS A LA RESOLUCIÓN DE PROBLEMAS}

\section{DESCRIPTIVE ANALYSIS PROTOCOL OF HEURISTIC STRATEGIES APPLIED TO PROBLEM SOLVING}

\begin{tabular}{|c|c|}
\hline PROPUESTA EDUCATIVA & $\begin{array}{l}\text { Carlos Minotta } \\
\text { Universidad de Antioquia (Colombia) }\end{array}$ \\
\hline Recibido:27/09/2014 & Aceptado: $17 / 02 / 2015$ \\
\hline RESUMEN & ABSTRACT \\
\hline $\begin{array}{l}\text { El presente artículo aborda el diseño de un } \\
\text { protocolo de análisis verbal que permita } \\
\text { identificar el tipo de operaciones cognitivas y } \\
\text { estrategias que una persona utiliza en la acción } \\
\text { de resolución de un problema, a su vez, permite } \\
\text { verificar el tipo de errores y sesgos en el } \\
\text { procesamiento de la información que dan lugar } \\
\text { a intentos fallidos de respuesta. Con el estudio } \\
\text { se propone construir un protocolo de } \\
\text { "pensamiento en voz alta" que facilite rastrear } \\
\text { de forma minuciosa, los múltiples procesos de } \\
\text { búsquedas de respuesta, y posibilite la } \\
\text { indagación y pesquisa de los patrones que son } \\
\text { las directrices subyacentes o reglas implícitas } \\
\text { bajo las cuales, un resolutor reorganiza los } \\
\text { elementos de un problema. La metodología } \\
\text { empleada fue la revisión documental derivada } \\
\text { en una propuesta metodológica de análisis. Se } \\
\text { concluye que el protocolo propuesto, posibilita } \\
\text { evaluar las vías alternativas de soluciones } \\
\text { plausibles y provisionales, que consisten en } \\
\text { intentos de solución experimentales derivados } \\
\text { de los yuxtapuestos y combinados sondeos de } \\
\text { patrones de pensamiento. } \\
\text { Descriptores: resolución de problemas, } \\
\text { estrategias heurísticas, pensamiento en voz alta. }\end{array}$ & $\begin{array}{l}\text { The present paper focuses on the design of a } \\
\text { verbal analysis protocol that allows the } \\
\text { identification of cognitive operations and } \\
\text { strategies used by a person in the action of } \\
\text { solving a problem; at the same time, it lets the } \\
\text { verification of some error and bias in the } \\
\text { processing of information that promote failed } \\
\text { attempts to answer. It is proposed to build a } \\
\text { "think aloud" protocol that facilitates the } \\
\text { detailed exploration of multiple processes for } \\
\text { search and answers and enables the pursuit for } \\
\text { patterns that are the underlying guidelines or } \\
\text { implicit rules under which a subject reorganize } \\
\text { the elements of a problem. The methodology } \\
\text { used was the documental review derived in an } \\
\text { analysis methodological proposal. It is } \\
\text { concluded that the proposed protocol helps to } \\
\text { evaluate alternative ways for plausible and } \\
\text { provisional solutions which are attempts for } \\
\text { experimental solutions coming from the } \\
\text { combined and juxtaposed surveys of thinking. }\end{array}$ \\
\hline
\end{tabular}

\footnotetext{
* Psicólogo. Universidad de Antioquia, Medellín (Colombia) psiquikam@hotmail.com.
} 


\section{INTRODUCCIÓN}

Si bien, es posible sostener como algo válido, la posibilidad de estudiar la estructura y el funcionamiento de las capacidades cognitivas de resolución de problemas, tanto o más pertinente, es conocer la manera como tales capacidades son puestas en práctica por los sujetos, es decir el proceso de pensamiento de análisis, síntesis y deducción, vistos ya no en términos generales o abstractos sino mas bien próximos a la experiencia, a través de la observación y examen de los mismos.

Resulta mucho más aprovechable explorar de forma minuciosa y en cada detalle los procesos de análisis y deducción, tal cual, como son llevados a la práctica por una persona particular, a la luz de un problema concreto.

Desde este punto de vista, toman relevancia; las ocurrencias únicas, la manera personal de disponer de los propios recursos de conocimiento, las preguntas y los medios originales -puestos al servicio de alcanzar el objetivo de hallar la respuesta a una incógnita,de los que hace uso una persona en particular. La búsqueda idiosincrática en la manera como cada quien busca los medios para encontrar la respuesta a un problema, puede definirse en términos particularizados, como por ejemplo, este intento, esta forma de hacerse preguntas, este problema, estos datos, estas preguntas, estas condiciones problemáticas, etc.

El presente estudio parte del supuesto de que la capacidad de descubrimiento y de invención de solución a un problema puede ser explicada a través de la exploración sistemática y exhaustiva de lo que las personas hacen con un problema; más específicamente, a través de la indagación de la pluralidad de métodos por las personas, cuando estas intentan resolver problemas. Incluso llega a considerar que es posible develar principios, reglas generales, encontrar constantes y regularidades, es decir, aquello que es común en las variadas estrategias heurísticas que las personas emplean.

Aquí vale la pena hacer una pequeña digresión respecto a lo dicho en el párrafo anterior, con el fin, de no caer en una aparente contradicción, en cuanto que, podría pensarse y no sin motivo, que las estrategias heurísticas concebidas por una persona, en tanto, le son singulares a ésta y obedecen a ocurrencias únicas y originales, no son generalizables, cierto es que, no es este el caso. 
De hecho, es posible encontrar un patrón recurrente y repetitivo en la manera como las personas abordan y resuelven los problemas a través de comparaciones interindividuales, e inter-grupales, por medio de, por ejemplo, el establecimiento de una categoría de "expertos" solucionadores de problemas y de "novatos" solucionadores de problemas.

Un gran número de estudios ha mostrado que los buenos resolutores de problemas se caracterizan por disponer de un conjunto de estrategias generales o heurísticas que guían su acción y que les ayudan a superar las dificultades que van encontrando durante el proceso de resolución. Estas formas de actuación son más o menos constantes en la resolución de problemas difíciles para el resolutor y en los cuales no se domina el contenido específico del problema (Manoli \& Jaume, 2001, p. 298).

Las estrategias heurísticas aplicadas a la resolución de un problema, son elementos que pueden ser aprehendidos, partiendo de ello, se pretende mostrar paso a paso, un método que permita el análisis de dichas estrategias, a saber, la manera como un experto, o novato en resolución de problemas se enfrenta a un interrogante.

El diseño del presente protocolo, tiene por propósito, la valoración de la forma como un sujeto desarrolla su proceso de análisis y hace deducciones, y por añadidura, propende por describir, los momentos de obstáculos y bloqueos, de descubrimientos y de hallazgos novedosos de soluciones. Permite identificar las reglas y pautas que hacen probable un resultado exitoso en la búsqueda de la solución a un problema, y encuentra las diferencias que tiene éste, con un intento fallido de respuesta, en función del análisis descriptivo que se haga de este proceso.

Para resumir, el interés que moviliza al presente escrito, puede sintetizarse en diseñar un instrumento capaz de resolver las siguientes preguntas ¿Cómo se desarrolla el proceso de pensamiento que emplea una persona para descubrir la respuesta a un problema de razonamiento lógico?, ¿Cuáles son las reglas o procedimientos cognitivos que hacen probable un acertado hallazgo o solución adecuada?

\section{Heurística}

El estudio de los métodos generales que conducen a la solución de problemas, así como el estudio del tipo de razonamiento que da lugar a dichos métodos, involucra los 
conceptos de heurística y algoritmo (Micha, 2003; Rosen, 2004; Alfaro, 2006). La heurística puede ser entendida como una capaciad cognoscitiva que consiste en generar vias o caminos de solución plausibles, de forma inmediata frente a problemáticas. El término "heurística" comparte la misma raíz etimológica que la palabra Eureka atribuida a Arquimedes, quien tras descubrir el principio que lleva su nombre, exclamó jeureka!, el cual puede significar <lo he encontrado〉, luego, así, la heurisitica se asocia a términos como descubrimiento, invención, o hallazgo. Para Polya, (1989, p. 101) la heuristica o la heurética, es una ciencia que tiene por objeto: "el estudio de las reglas y los metodos del descubrimiento y de la invención".

Polya (1989) además distinge una heuristica moderna que centra sus esfuerzos por: “comprender el método que se ocupa de la solución de problemas, en particular las operaciones mentales típicamente útiles en este proceso" (p. 102). La heurística así pensada estaría íntimamente ligada a la experiencia que se obtiene del enfrentamiento con numerosos problemas, y de la observación de los métodos de solución encontrados a ellos. Por esta razón, la heurística moderna se ocupa de encontrar métodos comunes de solución, independientes a cualquier problema, partiendo del supuesto de que es posible hallar reglas generales aplicables a numerosos tipos de problemas, por esta razón, el énfasis recae, en el estudio de las operaciones mentales, es decir, un tipo de razonamiento que sea capaz de ser descrito y pueda transmitirse a través de la enseñanza. El interés radica pues, en sistematizar procedimientos; en un principio intuitivos, pero que, una vez, estudiados, detallados y desarrollados, pueden ser aplicablicados a una diversidad de problemas.

Por razonamiento heurístico Markiewicz \& Etchegaray (2006) entienden un tipo de razonamiento conjetural, provisional, lanzado hacia delante en pos de una solución, pero que sin embargo no posee ninguna certeza de acierto, más bien, su objetivo es descubrir la solución y para ello, se vale de suposiciones, e hipótesis, las cuales, en un momento posterior, contrasta. El término plausible hace referencia a lo que es posiblemente cierto o valido, y por tanto razonable, pero no determinante, y tampoco infalible. El carácter provisional del razonamiento plausible hace referencia a que es contrastable, es decir, es las conjeturas han der ser verificables y refutables (Markiewicz, M. E., 2004).

Si bien se le atribuye al matemático griego Papus, el inicio de un campo de estudio denominado <analyomenos> traducible como "el tesoro del análisis o el arte de resolver 
problemas" se considera al trabajo de Polya, como uno de los pioneros en formalizar la heurisitica como estudio científico de las reglas del descubrimiento (Llera, 2000).

Se denomina estrategía heurística, a un artificio o truco ingenioso dirigido a alcanzar una meta, dado que es aplicable a una amplia variedad de problemas, puede decirse que las estrategias heurísticas son en buena medida independiente del campo problemático (Ferreras, 1999).

Ahora bien, dentro de las estrategias heurísticas podemos diferenciar aquella cuya aplicación no requiere de conocimientos e información específica sobre el problema a abordar, su aplicación reviste de una completa autonomia frente al dominio del problema, es decir, que sus procedimientos de aplicación no difieren, son los mismos, se aplican de la misma manera, y en el mismo orden, cualquiera sea el problema a solucionar, estas estrategias suelen denominarse métodos 《<débiles〉> de solución (Garnham, A., \& Oakhill, J., 1994). También, habrán estategias heurísticas que únicamente podrán ser aplicadas a un reducido número de tipos de problemas, por ello, son mas específicas y de menor alcance o potencia heurística: no obstante, suelen ser mas efectivas a la hora de solucionar problemas, de ahí que se les denomine <<fuertes〉>.

\section{Diseño de un protocolo de análisis descriptivo de resolución de problemas}

El protocolo de pensamiento en voz alta, es un instrumento metodológico utilizado para recopilar datos cualitativos, a partir de la información ofrecida por un informante acerca de lo que él está pensando a medida que resuelve un problema. El participante trata de decir en voz alta las ideas que le vienen a la mente, mientras tanto, el investigador utiliza algún medio para grabar las manifestaciones del participante, como por ejemplo una grabadora. Luego, el contenido de audio se transcribe y una vez transcrito se analiza con la ayuda de unas categorías de análisis previamente definidas (Castells, 2007).

Se pueden distinguir tres niveles de profundidad en los informes orales, de acuerdo al grado de esfuerzo de recuperación en la memoria de efectuar el informante. En el informe de nivel I, el participante dice de manera directa lo que piensa, valiéndose para ello, del contenido de información habido en su memoria de trabajo. En el nivel II, el participante agrega explicaciones, da detalles complementarios y esclarece el material verbal por él aportado, describiéndolo con mayor detalle y profundidad, ello puede llevar a un 
enlentecimiento de la tarea. Finalmente en el nivel III, el informante se ve exhortado a realizar una búsqueda de información en su memoria de largo plazo, es decir, explorar contenidos subyacentes no habidos en la memoria de trabajo, sino localizados en estratos más profundos, lo que implicaría un esfuerzo extra, a saber, un mayor uso de la capacidad de introspección del informante (Castells, 2007).

El diseño del protocolo de evaluación de la presente investigación muestra un nivel descriptivo, en tanto plantea de manera detallada el método estratégico que los participantes utilizan para resolver problemas; a partir del registro de la manifestación verbal de sus procesos de razonamiento. Dicho de otra forma, lo que se busca es observar cómo se efectúa el proceso de resolución de problemas a través del procedimiento de hacer explícitas las operaciones lógicas efectuadas que subyacen a la ejecución escrita. Se pretende con el instrumento; recolectar datos sobre las características de las variables componentes de la operación mental al resolver un problema, por ello se recopilan los aspectos y las dimensiones más relevantes de la resolución.

Esta herramienta de recolección de información (Análisis de protocolos verbales) se utiliza como instrumento de investigación (Frías, J. A, 2005; Brito, D. A., 2006) y ha sido utilizada en estudios de carácter experimental, en distintas disciplinas y ciencias, tales como la inteligencia artificial, y el diseño de sistemas expertos que simulan la forma como las personas resuelven problemas. El análisis de protocolo también ha sido utilizado por la psicología cognitiva, especialmente y de manera amplia en investigaciones sobre resolución de problemas.

Por medio de ésta técnica se insta a la persona a que "piense en voz alta" sus procesos de pensamiento mientras realiza una tarea, como por ejemplo, resolver un problema. Más específicamente, es preciso decir que el análisis de protocolo permite inferir los procesos cognitivos implicados en el trascurso de la resolución de un problema a través del registro sistemático de las acciones y palabras emitidas por la persona, esto es, a partir de lo que dice que piensa, de lo que recuerda, distingue, dice que siente, o percibe.

Se analiza entonces, por medio de la producción y expresión verbal, el contenido de los procesos mentales y para ello debe haber un registro fiel de la actividad realizada, lo cual se logra a través de un preciso encuadre sobre las condiciones necesarias de la 
entrevista. Esto es a lo que Frías, J., (2005.) denomina "registro, transcripción, codificación, segmentación y análisis" (p. 1).

$\mathrm{Al}$ investigar las operaciones discursivas nos interesaba saber, primeramente, cómo discurren en nuestros sujetos los procesos que son fundamentales para la resolución del problema (el análisis de los datos, elaboración de la hipótesis, el descubrimiento de los procedimientos para la resolución, la verificación de los resultados obtenidos a partir de los datos iníciales) (Luria, 1980, p.153).

La persona ha de expresar en voz alta su pensamiento, a través de comentarios e ideas que le surgen durante el trascurso de la tarea. Tales ocurrencias e hilo de ideas se convierten en datos para el investigador. Dentro de los principales inconvenientes de la utilización de ésta técnica, estriba el hecho de que el material recopilado está sujeto a la capacidad de introspección de la persona., entendiendo por introspección la posibilidad de observar dentro de sí, los propios procesos de pensamiento y tomar conciencia de ellos para poder hacerlos explícitos a través de la verbalización.

Según Ericsson y Simon citados por Garnham y Oakhill. (1994). "cuando el sujeto piensa en voz alta refleja lo que está ocurriendo en su memoria de trabajo a corto plazo" (p. 33). Sin embargo el mismo autor expresa que no hay consenso al momento de explicar cómo se relaciona exactamente lo que sucede en la mente de una persona y lo que dice. Referente a ello, Luria (1981) nos señala la dificultad habida en la indagación de la actividad intelectual y los procesos bajo los cuales ésta se manifiesta.

El análisis subjetivo del acto intelectual, que exige un desglose de la actividad en procesos intelectuales simultáneos, no da resultados seguros; su éxito es tanto mas fortuito cuanto que los procesos mentales se realizan habitualmente sin tener conciencia de ello, los intentos para describirlos por introspección a menudo sólo aportan conclusiones extremadamente pobres. (p. 10)

Aunado a lo anterior, dentro de las dificultades que surgen de la aplicación de un análisis de protocolo además de las ya mencionadas, se encuentran entre otras las señaladas por Aschcraft citado por Requena (2003), quien expone cuatro mecanismos cognitivos a tener en cuenta en la forma como el entrevistado procesa la información. Estos mecanismos son la automatización, aceleración, compilación, y distorsión; son tenidos en cuenta en el estudio del proceso de resolución para así, establecer la manera como a raíz de ellos, se 
afecta la recopilación de datos durante el trascurso de la ejecución de la tarea y la verbalización.

Automatización; gracias a la práctica los procesos mentales se hace cada vez más automáticos y por tanto inconscientes a lo largo de la vida de un individuo. -Aceleración; la ejecución se hace cada vez más rápida, lo que dificulta su registro. -Compilación; por efecto de la práctica las personas pueden realizar procesos cognitivos integradores de procesos más particulares (aquellos que interesan a la investigación). -Distorsión; la verbalización de los procesos mentales pueden distorsionar éstos o interrumpirlos.” (p. 4)

\section{Protocolo paso a paso}

A continuación se mostrará de manera amplia y pormenorizada, la estructura del diseño del protocolo de pensar en voz alta, y también la manera como ha de ser empleado para el análisis y descripción del proceso de resolución de problemas. Adicionalmente, se expone en detalle, el esquema general de identificación de estrategias heurísticas y el método de análisis que permitirá caracterizarlas. Valga precisar en términos metafóricos, que los procesos de pensamiento y comportamiento ejecutados por el informante, se consideran análogos a programas informáticos, por está razón, el lector advertirá, que de cuando en vez, se utilizarán expresiones propias del campo de la simulación del pensamiento por computadora, ello debido a que el presente protocolo se ampara predominantemente en el enfoque de la teoría del procesamiento de la información de la resolución de problemas de Newell \& Simon (1972). Para finalizar, se recomienda utilizar como material de estudio problemas bien estructurados, cuyo punto de partida y de llegada sean manifiestos con precisión.

Establecimiento de los objetivos: plantear de la manera más clara y precisa posible; los objetivos que se pretenden lograr con la realización de los reportes verbales, es decir, definir de antemano el tipo de conocimiento que se proyecta obtener especificando el contenido del proceso mental o atributo latente a evaluar. Los objetivos deben ser taxativos, concretos, específicos y factibles. Para el caso que nos atañe, el protocolo aquí presentado, se diseña con el objetivo de: Identificar estrategias heurísticas, y procedimientos algorítmicos en la resolución de problemas. 
Metodología: Estudio de caso: se resuelve diseñar el protocolo de forma tal, que se constituya en un estudio de caso, esto por varias razones además de las inicialmente mencionadas. A saber, el estudio de casos es una metodología de indagación que permite al investigador, llevar a cabo, un examen extenso y detallado, sobre múltiples aspectos de un mismo sujeto u objeto concreto (Lopez Parra, 2002).

Aparte de lo anterior, el estudio de caso hace uso de la evidencia surgida del contraste de la teoría con los hechos, acumulando y examinando pruebas empíricas cuyo valor de dato es verificable y refutable, encima de ello, basa su aplicación en un razonamiento exploratorio e inductivo tendiente a generar conocimientos generales que partan de la observación de casos particulares y la comprobación lógica de conjeturas al ponerlas a prueba con los datos, y viceversa.

Reexamina la información recopilada a partir de la cual se construyeron las hipótesis, con la finalidad de encontrar relaciones no vistas, o inferir otros patrones y regularidades en la lógica de articulación de los datos. Además, el estudio de casos permite abordar al sujeto u objeto de estudio desde múltiples perspectivas, de esta manera, es posible hacer converger y contrastar puntos de vista disimiles (teorías) en un mismo aspecto del objeto a investigar (Lopez Parra, 2002), para el caso que aquí concierne: la transcripción del protocolo.

Instrumentos de recolección de información: Grabadora de audio; se emplea para el registro auditivo de la verbalización del informante.

Se hace uso de la entrevista semiestructurada, dado que no se realizan preguntas en un orden predefinido ni prefijado, en el transcurso de la entrevista se irán planteando las preguntas acorde al ritmo del participante y según su manera idiosincrática de resolver el problema. A su vez se procurará que las preguntas sean abiertas y generales para que así no induzcan la respuesta.

Las preguntas pueden ser formuladas; tanto en tiempo pasado ¿Cómo te surgió esa idea?, como en tiempo presente ¿cuál crees que es la solución y por qué? Por último, téngase en cuenta que, las preguntas han de formularse siempre y cuando el investigador considere que su respuesta es clave para el reporte, o cuando se produzca en el informante 
un silencio prolongado, de no darse ninguno de los casos anteriores, se recomienda en lo posible no hacer uso de la guía de preguntas de la entrevista.

En general se exhorta a que no haya ningún tipo de injerencias por parte del investigador en el transcurso de la resolución al problema, cada pregunta lanzada al informante se considera una intervención, se presupone que entre menos intervenciones se realicen; mayor grado de fidelidad posee el registro del protocolo para con los procesos de pensamiento del informante.

Si bien, puede afirmarse que resolver un problema es un proceso mental complejo e indivisible y en el cual confluyen numerosos factores mutuamente interdependientes, para efecto de su descripción será necesario fragmentar el proceso en diferentes momentos (análisis) para así, luego reconstruirlo interpretativamente.

Criterios de selección de participantes: se recomienda que la persona decidida a presentar su colaboración en un análisis de protocolo, (1) esté en capacidad para reportar verbalmente sus procesos de pensamiento a la par que ejecuta una tarea. (2) tenga capacidad para seguir instrucciones (Renquena, 2003). Y (3), esté motivada y con interés en participar (Brito, D. A., 2006). Por otra parte, la selección de los participantes puede hacerse por el área de desempeño y conocimientos involucrados en el estudio, de ser así, pueden elegirse personas con destrezas comprobadas en el área a investigar o expertos, según sea el objetivo de la investigación.

Por otra parte, en lo que atañe al protocolo aquí propuesto, cabe señalar que se diseña pensándolo para ser aplicado a uno o a pocos participantes, dado el grado de exigencia que implica la realización de cada una de las etapas del mismo, a saber, entrevista, transcripción, análisis e interpretación de datos, lo cual demanda un dispendioso tiempo, sin contar; con la previa revisión de literatura, documentación y adquisición de amplio conocimiento y dominio sobre el tema alrededor del cual gira la realización del protocolo.

Encuadre: consiste en la comunicación al informante de las condiciones y reglas con las cuales se llevará a cabo la realización del protocolo, en este momento, se le detallan las instrucciones que debe seguir tales como, intentar resolver el problema presentado y 
'pensar en voz alta', es decir, expresar cada una de sus ideas referidas a lo que está haciendo.

De forma simultánea se le pide al participante efectuar la lectura del problema y disponer de bolígrafos y hojas de papel si así lo solicitase Se le expone el propósito de la investigación y en específico, los objetivos que se pretenden con la misma, en un lenguaje sencillo y accesible.

Acto seguido, se le comunica al participante acerca de los instrumentos que acompañarán al reporte verbal, estos son; una grabadora de audio, un dispositivo de grabación audiovisual, informándole que son herramientas indispensables para un registro fiable de su verbalización, a su vez, se le explica de antemano, el hecho de que no hay una forma correcta o modo incorrecto de resolver el problema, lo cual es verídico, hasta cierto punto, puesto que de todas formas habrán respuestas acertadas al problema y otras, por el contrario, no podrán considerarse acertadas, sea porque infringieron algunas condiciones del problema o porque definitivamente no satisfacen la incógnita.

El investigador conociendo de antemano la respuesta - cosa muy aconsejable, de hecho sugerible- podrá de forma excepcional, frente a errores e inconsistencias de la respuesta aportada por el participante; utilizar para con el/ella la técnica del descubrimiento guiado, o confrontarle de forma directa exponiendo una inconstancia evidente en el razonamiento arrojadora una respuesta equívoca. Finalmente, el encuadre ha de acompañarse del requerimiento ético del consentimiento informado.

Prueba piloto: se efectúa con varios propósitos, uno de ellos es, el de familiarizar al participante con el procedimiento de recogida de información, de modo que pueda habituarse a la situación de ser observado mientras efectúa una tarea, también la prueba se realiza con el intención de verificar si el informante ha comprendido; sí o no, a cabalidad las instrucciones dadas.

Registro y sistematización: esta etapa de la realización del protocolo, se divide en dos momentos, el primero consiste en el registro verbal propiamente dicho, durante su transcurso, el investigador conduce la entrevista, hace anotaciones, y toma apuntes. 
En un segundo momento se acomete la transcripción del registro almacenado en formato digital. La versión escrita del registro de audio se acompaña en su realización, de una serie de escollos que complican dicha tarea, a saber; por una parte, el investigador ha de estar atento a las inflexiones de la voz, en ocasiones tendrá que vérselas con la exigencia de atinar en la elucidación de una frase o palabra ininteligible, así mismo ha de adecuarse al ritmo, escogencia de palabras y pronunciación del informante (Montero \& Farías, 2005) y a su vez, tendrá que hacer uso de manera inevitable de sus facultades interpretativas lo cual trae implícito, una ineludible reconstrucción del relato del informante, esto se haceevidente cuando el investigador de forma deliberada; decide qué tramos de la cinta son rescatables y cuáles no, lo cual refleja el efecto selectivo de la atención y el imperativo de focalizar los esfuerzos en hacer una reconstrucción coherente de lo registrado auditivamente, así ello, implique borrar de la versión escrita fragmentos del audio; en función de la fabricación de un escrito entendible para el investigador.

Tomando en cuenta, las consideraciones aquí señaladas, se proseguirá a exponerse en detalle algunas pautas específicas y recomendaciones útiles para el registro sistematizado de los datos:

La transcripción de la verbalización del informante, ha de llevarse a cabo en distintos formatos acorde al nivel de análisis que se requiera. Primeramente, se procederá a transcribir el relato en un pliego en blanco, de este modo, la transcripción discurra de manera continua. Aquí conviene llamar la atención sobre un aspecto de suma importancia, a saber, la utilización de los signos de puntación, en particular de aquellos que son indicativos de pausas y determinan el significado de una frase, entre estos signos se destacan, el punto, la coma y los dos puntos.

Por otra parte, recuérdese transcribir igualmente las figuras, fórmulas y todo lo notado por el informante en la hoja de apoyo que se le dispensó. Con respecto a esto último; téngase en cuenta la posibilidad de recortar el tramo de espacio que ocupa la figura en la hoja de apoyo, y adherirla a la hoja de registro, en caso dado de que se dificulte realizar una copia semejante -no necesariamente exacta- de la figura.

Procúrese igualmente, conservar la sincronización entre lo dicho y lo escrito, por el informante, es decir, que las fórmulas, figuras, trazos o dibujos han de ir acompañados de 
Protocolo de análisis descriptivo de estrategias heurísticas aplicadas a la resolución de problemas Minotta Carlos (pp. 95-118)

las frases y tramos del relato, con los cuales están relacionados o fueron concomitantes en el tiempo.

Volviendo a las características del primer pliego, éste debe contener en el margen superior izquierdo; la formulación del problema y en el margen superior derecho la siguiente información: la fecha y hora de realización del registro audiovisual y el seudónimo del informante. Para mayor ilustración véase el siguiente modelo:

Problema: los puntos $A, B, C$, y $D$ forman un cuadrado, cuyo $\quad$ Día, mes, año:

lado es $2 \mathrm{~cm}$, halle el perímetro de la región sombreada. Hora:

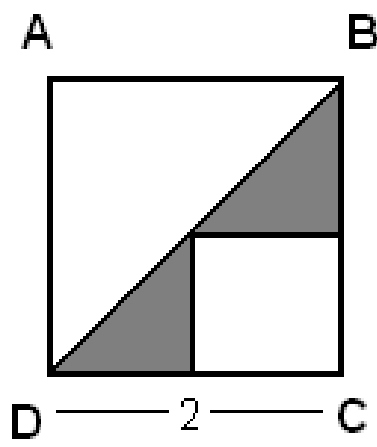

Nombre:

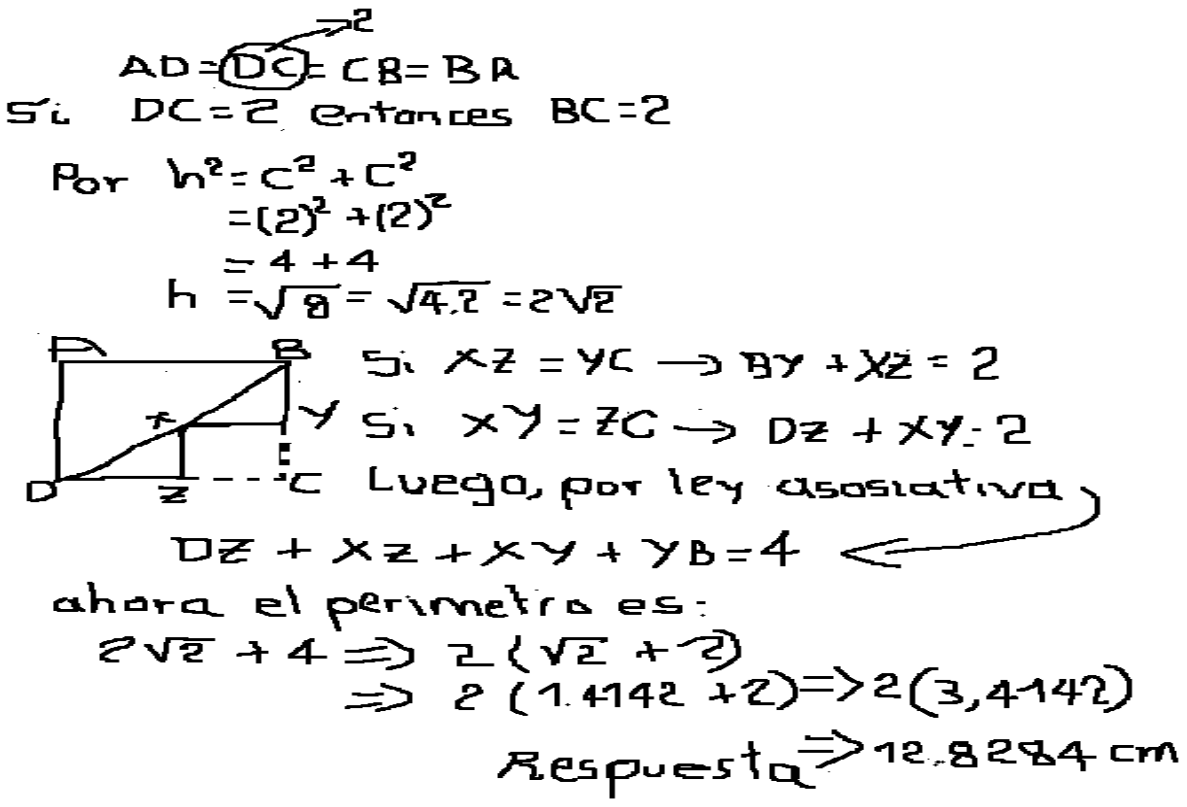

Figura 1. Pliego $1^{\circ}$ del registro del protocolo 
Seguidamente, la transcripción se reproducirá en un segundo pliego numerado en sentido vertical, para formar con ello, una columna que luego será fraccionada por filas que interceptadas con los extremos de las columnas formarán celdas.

A cada celda le corresponde un número, asignado en orden consecutivo y ascendente, es decir se enumerará el pliego de arriba abajo, comenzando por la celda del extremo izquierdo. Luego se inserta al margen derecho de la numeración otra columna amplia en extensión con igual número de filas. Las celdas enumeradas albergan como función, el fraccionamiento del universo de información transcrita, por consiguiente, constituyen la mínima unidad básica de análisis, en ellas, se alojarán segmentos de texto, referidos a expresiones, frases u oraciones; entendiendo por frase: un curso de palabras cuyo conjunto da lugar a una unidad y por oración: la menor unidad del habla que posee un sentido completo. Así:

\begin{tabular}{|l|l|}
\hline & \multicolumn{1}{|c|}{ Contenido } \\
\hline 1. & \\
\hline 2. & \\
\hline 3. & \\
\hline 4. & \\
\hline 5. & \\
\hline 6. & \\
\hline 7. & \\
\hline 8. & \\
\hline$\# \ldots$ & \\
\hline &
\end{tabular}

Figura 2. Pliego $2^{\circ}$ del registro del protocolo

A la vez, las celdas pueden contener información tipo variable y/o constante, el primer tipo consta de datos expresados en términos de fórmulas o ecuaciones cuyos miembros son cantidades desconocidas, entre ellas; la incógnita, habilitadas para asumir cualquier valor, mientras que los datos constantes, poseen valores específicos y conocidos.

Posteriormente, el contenido fraccionado del segundo pliego, pasará a constituir en un tercer pliego; una matriz de datos, (por matriz entiéndase una tabla cuyo espacio se haya 
distribuido únicamente en un plano de dos dimensiones); a lo largo y a lo ancho. La utilidad de una matriz radica en la facilidad que comporta para registrar y relacionar datos ordenándolos en filas y columnas, cuya intercepciones forman celdas, como ya se ha dicho, una celda es la resultante de una intercepción entre el espacio de líneas paralelas horizontales (filas), y el espacio entre las líneas verticales (columnas). En este caso en particular, cada fila constará de series de celdas que conservarán su correspondiente número de identificación o de registro otorgado en el segundo pliego.

No hay un límite de restricción para la prolongación de las dimensiones de la matriz, su extensión estará dada por el número de filas y columnas. Se espera que el carácter bidimensional, cuadriculado y rectangular de la matriz facilite una representación cartográfica del proceso de resolución de problemas.

Un conjunto de varias celdas, forma rangos de celdas (agrupaciones) cuya composición da lugar a las operaciones realizadas por el informante, y a descripciones de estados intermedios de conocimientos. Ejemplo:

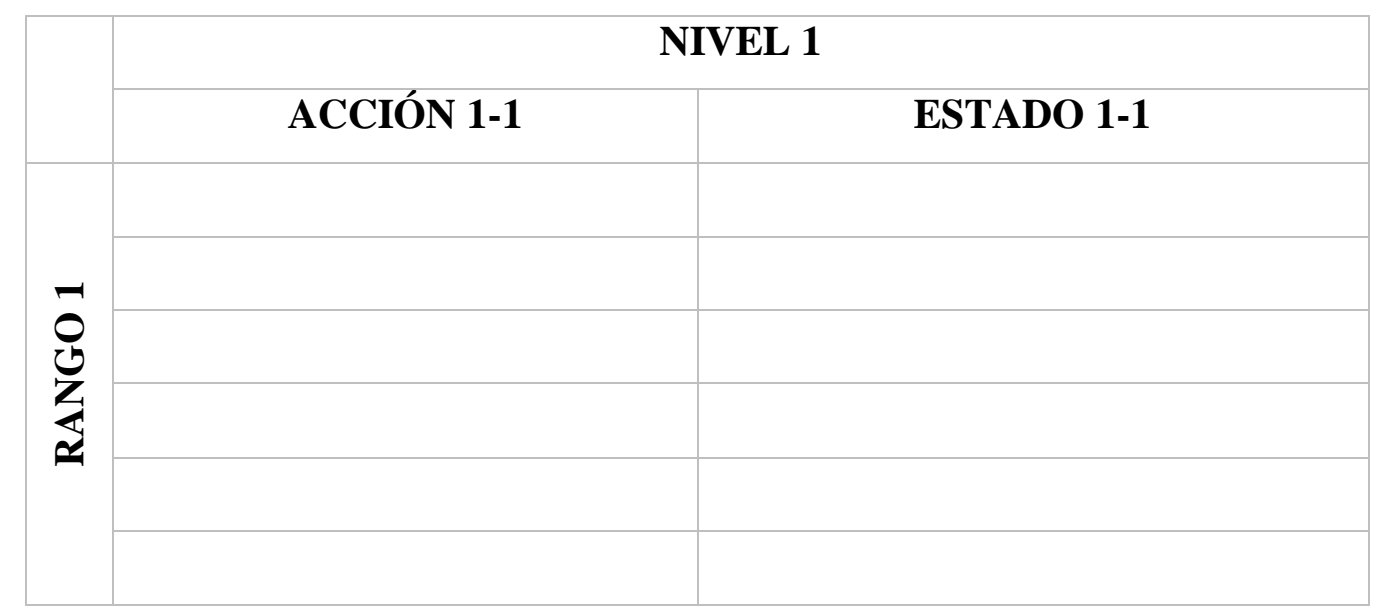

Figura 3. Matriz de Datos

Cuando una persona realiza una acción, el mundo del problema cambia de un estado a otro. De esta manera, el proceso de resolución comprendería dos tipos de secuencias; las de procedimientos u operaciones efectuadas con vista a hallar la solución y las secuencias de estados o configuración en la disposición y arreglo de las partes del problema que deriven de cada operación. 
De los párrafos anteriores se deduce que una celda contiene valor de dato, en tanto es la unidad mínima de significado en el proceso de registro. Sin embargo, de ninguna manera se considere a las celdas: unidades aisladas, antes por el contrario, cada una se halla en relación con otra,

Dicho en otras palabras, en ocasiones el contenido de una celda corresponde a una proposición que no posee autonomía semántica o carece de independencia, puesto que su sentido es dependiente de una proposición precedente. Cuando ello sucede, una proposición ulterior a otra, se haya subordinada a la primera, a través de una conexión lógica; (entendiéndose por proposición una entidad gramatical que constituye la unidad de significado inmersa en una oración, y que por tanto, le otorga el contenido lógico a la misma).

Partiendo de la observación anterior, se concluye como algo factible, que una misma operación ejecutada por el informante pueda requerir de una agrupación de varias celdas para poder ser descrita, y es lo mismo para un estado intermedio. Una vez se combinan las celdas, éstas dan lugar a la representación completa de una acción. Siendo igualmente factible, que una misma celda pueda contener una proposición participe de la descripción de dos operaciones diferentes, es decir que complemente el significado de distintas celdas de manera simultánea.

Algo similar sucede con la lectura de cualquier texto, si una persona se dispone a leer un libro, y toma al azar una página cualquiera y de esa página lee una oración (a), difícilmente podrá entender el significado de la frase leída, si no lee también, la frase anterior (b) y la que le antecedía a esta última, o sea (c), y así sucesivamente. La serie de frases (a), (b), (c)..., tomadas cada una de forma aislada no da lugar a un significado completo. Es la suma ordenada de ellas $\{(a)+(b)+(c) \ldots\}$, aquello que ofrece el sentido cabal de una acción realizada o la descripción de un estado de cosas. A propósito Renquena (2003) señala:

Si durante la codificación, el investigador se da cuenta que algún grupo de segmentos consecutivos refieren un procedimiento, paso o estrategia global en la realización de la tarea, aquél puede efectuar también una codificación sintética: interpretar el grupo como un todo, no sus elementos. Inversamente, puede ocurrir que un mismo segmento sea la expresión simultánea de más de un proceso; en tal caso, el investigador codifica el segmento de manera múltiple, indicando que pertenece a varias categorías. (p.12) 
A continuación, se ilustrará un extracto del formato del tercer pliego de registro acorde con las indicaciones ya señaladas. Con el siguiente esquema gráfico se procura obtener una representación en serie y en paralelo de la secuencia de operadores y de los distintos estados que conforman el espacio del problema.

\begin{tabular}{|c|c|c|c|c|c|c|c|c|c|}
\hline & & FL 1 & & & FL 2 & & & IEI 3 & \\
\hline & ACCIÓN 1-1 & ESTADO 1-1 & & ACCIÓN 1-2 & ESTADO 1-2 & & ACCIÓN 1-3 & ESTADO 1-3 & \\
\hline & & & & & & & & & \\
\hline o & & & o & & & o & & & 0 \\
\hline $\bar{z}$ & & & $\bar{z}$ & & & $\bar{z}$ & & & $z$ \\
\hline & & & $\approx$ & & & $\approx$ & & & \\
\hline & & EL 1 & & & EL 2 & & & EL 3 & \\
\hline & ACCIÓN 2-1 & ESTADO 2-1 & & ACCIÓN 2-2 & ESTADO 2-2 & & ACCIÓN 2-3 & ESTADO 2-3 & \\
\hline$N$ & & & $\mathrm{~N}$ & & & N & & & \\
\hline ఫ్ర & & & O & & & \& & & & O \\
\hline Z & & & z & & & z & & & z \\
\hline & & & & & & & & & \\
\hline & & EL 1 & & & EL 2 & & & EL 3 & \\
\hline & ACCIÓN 3-1 & ESTADO 3-1 & & ACCIÓN 3-2 & ESTADO 3-2 & & ACCIÓN 3-3 & ESTADO 3-3 & \\
\hline 2 & & & $m$ & & & $m$ & & & \\
\hline D & & & D్ & & & D્ & & & ్ְర \\
\hline$\underline{5}$ & & & 4 & & & $\leq$ & & & 3 \\
\hline & & & & & & & & & \\
\hline & & EL 1 & & & EL 2 & & & EL 3 & \\
\hline & ACCIÓN 4-1 & ESTADO 4-1 & & ACCIÓN 4-2 & ESTADO 4-2 & & ACCIÓN 4-3 & ESTADO 4-3 & \\
\hline 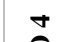 & & & + & & & I & & & \\
\hline SO & & & D & & & D & & & O \\
\hline ב & & & $\frac{1}{x}$ & & & 5 & & & 5 \\
\hline & & & & & & & & & \\
\hline & & EL 1 & & & EL 2 & & & EL 3 & \\
\hline & ACCIÓN 5-1 & ESTADO 5-1 & & ACCIÓN 5-2 & ESTADO 5-2 & & ACCIÓN 5-3 & ESTADO 5-3 & \\
\hline & & & in & & & & & & \\
\hline రి & & & D. & & & D્ & & & ర్d \\
\hline$\approx$ & & & $\widehat{x}$ & & & $\underline{\approx}$ & & & $\underline{z}$ \\
\hline & & & & & & & & & \\
\hline
\end{tabular}

Figura 4. Pliego $3^{\circ}$ del registro del protocolo

Los niveles fraccionados por cada rango surgido de la agrupación de varias filas, están demarcados por la asociación de dos columnas, una de ellas referida a una acción y la otra a un estado de conocimiento. A su vez, tanto los operadores como los estados, están compuestos por la asociación de varias celdas, esto es así porque -como ya se había 
mencionado- el valor de dato de cada una de ellas se haya en relación con el valor de dato de otra para dar lugar a una acción integrada o a un estado de conocimiento completo.

Los niveles aumentan de izquierda a derecha y describen el grado de avance o progreso general obtenido por la persona en su búsqueda de solución a un problema, entretanto, los rangos acrecientan su numeración de arriba-abajo y representan saltos cualitativos en la dirección de búsqueda, que indican que el solucionador dirige su secuencia de operadores a un objetivo distinto. Usualmente las razones para que se produzca dicho cambio son las siguientes:

En prime lugar: el solucionador se ha percatado de que el plan llevado a cabo, terminará dando un resultado infructuoso, por lo cual, decide reformularlo parcial o totalmente, en éste último caso, abandona el plan estratégico de solución y diseña otro, lo cual se corresponde con una nueva secuencia de acciones-estados en un rango distinto. Si el plan se reformula totalmente, la nueva cadena de acciones inicia desde el primer nivel pero en el rango subsiguiente, mas si aconteciere que el plan se varió únicamente en parte, entonces, una nueva secuencia de operadores-estados empezará desde el nivel que alberge un estado similar al estado creado desde la aplicación del nuevo primer operador que conforma el plan estratégico de solución diseñado de manera alterna al primero; que ahora considérese fallido.

El proceso aquí señalado, referido a la reformulación de la estrategia, puede repetirse variadas veces, por lo que, a cada reformulación le sigue una nueva secuencia de operadores; secuencias ubicadas en tantos rangos como reformulaciones halla. Por ejemplo, si una persona en el transcurso de su solución a un problema, se encontrase en el estado (13) producido a partir del operador (1-3), y se diese cuenta de que ha entrado a un callejón sin salida, puede decidir, volver a las condiciones iníciales del problema, y a partir de una nueva consideración de los elementos del mismo, elaborar una nueva estrategia, cuyo primer operador sería (2-1), el número dos indicaría el desarrollo de una secuencia de movimientos diferente a la inicial. Igualmente, podría acaecer, que dentro del plan original se modificase únicamente la última operación realizada, a saber, (1-3) -porque a juicio del solucionador, tal operación conllevaba un error- y en su lugar, se ejecutase una acción distinta, con respecto al mismo estado de conocimiento (1-2) al cual le fue aplicado la operación (1-3), dicha nueva acción aunque correspondería al nivel 3, formaría parte del 
segundo rango, por lo que se expresará con el número de registro (2-3) y sería descrita por las celdas que componen su respectivo campo.

En segundo lugar: habrán problemas que por sus mismas características requieran la vuelta a estados de conocimiento anteriores, en dichos problemas, es posible constatar que el solucionador se verá eventualmente en la necesidad de retroceder a un estado pretérito y alejarse temporalmente de la meta, aumentando la distancia para con ella. Lo anterior se puede representar en la estructura de datos, adicionando un nuevo estado: con la descripción de la configuración de los elementos de un estado precedente, en el mismo nivel en el que dicho estado precedente se encuentra, pero en el rango inmediatamente subsecuente, de esta manera la matriz reflejará una aparente regresión en el progreso general de la solución, aparente porque, aunque ha se ha efectuado una transición a un estado anterior, ha habido sin embargo un cambio de rango.

En tercer lugar, habrá problemas frente a los cuales una persona puede emplear el heurístico de análisis de medios y fines, en tal caso, cada rango contendrá secuencias de operaciones dirigidas a alcanzar pequeñas sub-metas. De esto se infiere que la aplicación del heurístico de subir la cuesta, dará como resultado una matriz de datos de un rango, puesto que, con dicho heurístico se sigue una sola pauta de acción, de suerte que el solucionador transita en una única ruta de principio a fin.

Por último conviene aclarar que el modelo cartográfico presentado en el pliego número tres, es flexible en cuanto al número de celdas permitidas por rango, en su efecto, es el investigador quien toma la decisión de fijar estimativamente; cuántas celdas incluir por cada rango, eso, claro está, sea acorde a las características del problema estudiado, al estilo, al modo de habla, lacónico, o abierto y a la eficacia del solucionador, para así en base a ello, estimar aproximativamente el número de celdas a insertar en los rangos, sin embargo, valga precisar que en todo caso, una vez transcrito la totalidad del contenido, se ha de iniciar una poda de celdas, esto es, se eliminarán las celdas en blanco, sea; marcándolas, cortándolas, u oscureciéndolas, con motivo de facilitar el análisis del perfil de la información contenida.

Toda vez que, el pliego una vez recortado, muestra el camino recorrido por el solucionador, las celdas en blanco son marcadas o apartadas del pliego, no porque, no sean útiles, al contrario, si por una parte, las celdas ocupadas, dejan advertir las elecciones 
estratégicas, las acciones tácticas, y las múltiples selecciones de opciones de actuación, entre cuantiosas alternativas, (todas en principio posibles), las celdas en blanco, dan cuenta, por su parte, del recorrido opcional, y acciones potenciales que el solucionador pudo en determinado momento; llevar a cabo y en definitiva no hizo, ya sea porque no las concibió (en cuyo caso, la falta de un tipo específico de conocimiento, limitó; su margen de libertad y de acción) o porque, aún, habiendo percibido, una vía, -sea acertada o no, de solución)- , en últimas, el solucionador optó, por aquel curso de acción, materializado en sus actos. Aquí expresa, entonces, la característica definitoria del tercer pliego de registro, aquello que la construye, son básica y fundamentalmente; las elecciones consumadas por un sujeto, sometido a un proceso incesante, de toma de decisiones.

Decisión: todo comportamiento implica una selección, consciente o inconscientemente, de determinadas acciones, entre todas las que son físicamente posibles. - existe, en todo momento, una multitud de alternativas posibles, cualquiera de las cuales puede ser emprendida por un individuo determinado, estas numerosas alternativas quedan reducidas, por algún proceso, a aquella que, en realidad es llevada a cabo. (Simon, 1972, p. 5)

\section{Método de análisis}

Se desarrolla un esquema de análisis que permita describir las estrategias heurísticas desde una perspectiva molar y otra molecular. El nivel de análisis puede variar acorde a las características de la estrategia a identificar, más propiamente en lo concerniente al grado de especificidad o concreción de la misma, ella (la estrategia) puede ser lo suficientemente específica como para definirse en un único movimiento y ocupar tan solo una celda en la hoja de registro, de igual modo pudiese ocurrir lo inverso; a saber, que una estrategia sea lo bastantemente compleja como para requerir en su descripción toda una secuencia ordenada de movimientos, o lo que es lo mismo, ser definida a partir de un conjunto de procedimientos.

En el primer caso, la estrategia heurística está comprendida por una única acción, sea física o mental, bien definida, discreta e indivisible, mas no así en el segundo caso, en éste, la estrategia aparece compuesta de una agrupación de múltiples acciones superpuestas y organizadas dinámicamente, sucedidas de forma secuencial, e interconectadas según los 
parámetros de un principio ordenador que unifica las acciones y las controla orientándolas a un objetivo.

Ahora bien, cabe señalar que la integración y unificación de los movimientos que componen una estrategia general se realiza solo en parte, ergo cada acción comprende un sub-objetivo por cumplir que se haya subordinado al objetivo primordial que es solucionar el problema. Por otra parte, a medida que la estructura de la estrategia heurística aumenta en su complejidad, lo hace también la especificidad de cada una de las acciones que la conforman.

En síntesis, la estrategia heurística lejos de ser una acción indiferenciada o difusa, es por el contrario una organización jerárquica y descentralizada de operaciones con diversos alcances, operaciones que si bien, se subordinan unas a otras, guardan cierta autonomía; la suficiente como para dirigirse a un propósito específico, e incluso modificar, de ser necesario, el plan general de acción o la estrategia global. Ello debido al carácter heterárquico de la misma, es decir, que la aplicación de un operador de menor alcance puede develar en el transcurso del proceso de solución una falla o inconsistencia en el plan general, obligando al solucionador a replantearlo en parte o en su totalidad. En consecuencia, la función de control entre la estrategia general y las operaciones específicas no es unidireccional sino recíproca. Lo cual se revierte a su vez, en una mayor flexibilidad adaptativa hacia las exigencias de una tarea o problema en particular.

\section{Análisis de representaciones estado-acción}

Cuando un sujeto es desafiado por un problema, es sumergido en un mundo perplejo, cuya situación o estado plantea baches e información incompleta. En aras de salir airoso de dicho estado de cosas, el sujeto se ve en la necesidad de realizar acciones que movilicen la configuración de los elementos al interior del mundo, esperando con ello, que el estado del mundo se modifique en su estructura. Se denomina operadores a las acciones realizadas por el sujeto que son capaces de transformar el estado enredado del mundo en otro mucho más completo y resuelto (Garnham, A. \& Oakhill, J., 1994).

Un método eficaz para describir el patrón de comportamiento de una persona inmersa en un problema, es caracterizar su actuación como un proceso de búsqueda de respuestas con miras a salir de la colección de estados intermedios creados al interior del espacio del 
problema, lo cual se logra dándole una solución al mismo. De esta manera, la forma gráfica de esquematizar el proceso de resolución de un problema es describiendo la secuencia de operaciones, conforme al orden en que éstas son realizadas por el sujeto, y también describiendo la secuencia de estados resultantes con la aplicación de cada operador.

El esquema gráfico de representación del par binomio acción-estado, se facilita en aquellos problemas bien estructurados en los cuales los estados inicial y final están claramente definidos y sin ambigüedad, al igual que las acciones legales, es decir, aquellas que pueden ser ejecutadas por la persona y que no infringen las condiciones del problema. Puede ser que, en algunos problemas, los operadores estén descritos en el enunciado, de no ser así, entonces se deduce que están implícitos o formulados de manera tácita, por lo cual, son susceptibles de inferirse.

A medida que el solucionador se desplaza de un estado a otro, realiza un proceso de búsqueda de solución, utilizando los operadores disponibles en su memoria que sean útiles para salir del estado del mundo en que se encuentra, luego selecciona el operador que considera más idóneo para dicha tarea, y lo mismo ocurre en el nuevo estado producido por la aplicación del operador, reiniciándose así el proceso de búsqueda. Lo expuesto en los párrafos precedentes hasta aquí, puede resumirse en las siguientes palabras de Garnham, A. \& Oakhill, J. (1994):

En cualquier momento, el mundo se encuentra en un estado particular, y existen cierto número de maneras en las que dicho estado puede alterarse. Un estado del mundo puede ser representado mediante un modelo mental de la parte relevante de dicho mundo, y las acciones (u operadores) se pueden representar como (métodos de realizar) transiciones de un estado del mundo a otro. (p.228)

\section{Una forma alterna de análisis. Arboles de representacion estado-acción}

Los distintas alternativas de movimientos elegibles para ser aplicados al estado actual, y el conjunto global de las elecciones totales que la persona llevó a cabo durante su recorrido por los diferentes estados, pueden ser representados a través de una estructura de datos que simula la fachada de un árbol, en ella se pueden graficar los movimientos que la persona realiza al igual que los estados por los cuales transita.

El árbol posee una raíz o punto de inicio ubicado en la parte superior, que representa el estado inicial del problema, de la raíz surgen ramas secundarias desprendidas de forma 
simultánea y ubicándose en extremos opuestos, ellas representan movimientos o acciones, de la punta de cada una se produce un punto que simboliza un nuevo estado creado a partir del movimiento. Luego nuevamente se produce una ramificación, esta vez, a partir de las ramas secundarias, dándose así; lugar a ramas terciarias con diferentes estados en su pico. Es decir, que la aplicación de un movimiento dio lugar a la existencia de un estado al cual se le pueden aplicar múltiples movimientos posibles.

La forma en términos de tamaño, o amplitud que adopte el árbol dependerá de la gama de alternativas de movimientos que la persona tenga para elegir, y los estados que se produzcan con dicha elección. Gama de alternativas que a su vez dependerá de la elección de un movimiento precedente. Los árboles en tanto, representación gráfica, permiten organizar de manera esquemática el proceso de resolución de problemas codificado en datos de operadores y estados.

Usualmente el árbol se ramifica de arriba abajo y en sentido descendente, de suerte que, el transcurso de la actividad de resolución queda reflejado a partir de un sistema de sucesión, esto es, que el conjunto de todos los movimientos se ordena acorde al orden en que fueron realizados, de esta manera, los movimientos ubicados arriba o cerca de la raíz fueron los primeros y los movimientos que se hallan debajo serían entonces, aquellos últimos que se realizaron, por consiguiente, una acción que anteceda a otra en el tiempo, se ubicaría arriba de ésta última.

El árbol también exhibe un sistema filial, en donde las acciones que posibilitan la emergencia de otras, se hayan unidas a estas últimas por medio de enlaces o nodos que representan los estados de conocimiento, que la primera acción creó y que las segundas a su vez tratan de cambiar o modificar en otro estado diferente. Añadido a todo lo demás, hay que precisar que las distintas ramificaciones se distribuyen por niveles, así, el nivel (1) estaría constituido por el estado inicial, el segundo nivel (2) comprendería la primer ramificación de las acciones posibles o alternativas aplicables al estado inicial y que dan lugar a distintos estados a su vez. Véase a continuación, el siguiente ejemplo de un árbol estado-acción. 


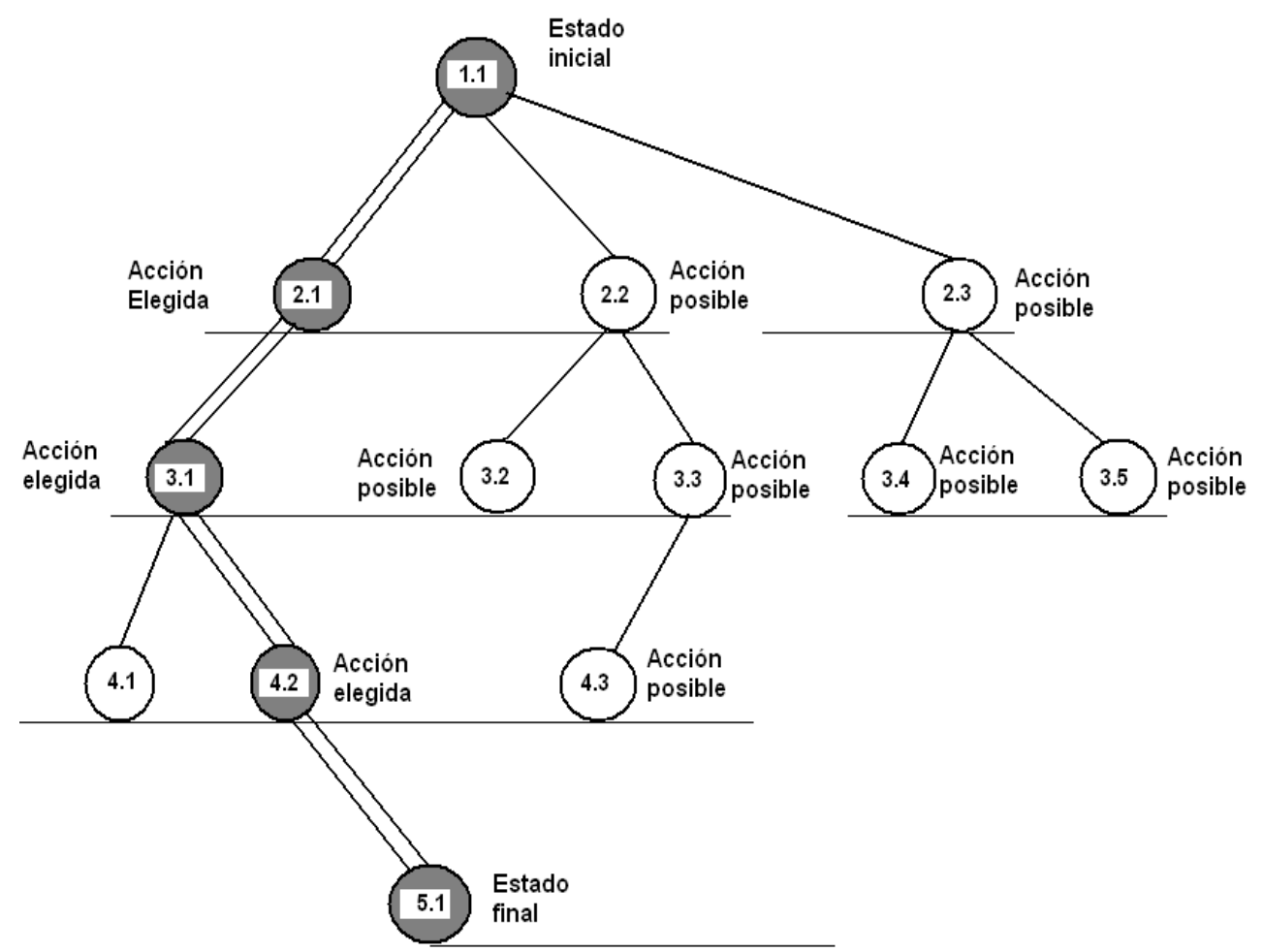

Gráfico 1. Proceso de solución a un problema

La anterior es una representación gráfica del proceso de solución a un problema, utilizando el diagrama: árbol estado-acción. Allí se muestran los diferentes estados que una persona construye con cada acción y por los cuales se desplaza en la búsqueda de la solución. Un círculo representa la acción que la persona puede realizar y a la vez, el estado producido por la ejecución de la misma, por otra parte, el número inscrito en el círculo indica el nivel de ramificación del árbol, y la identificación de cada acción con su respectivo estado.

De la observación de la gráfica se deducen cinco niveles desarrollados. Si de interpretar la gráfica se trata, obsérvese que la persona, en un primer momento, está en la capacidad de llevar a cabo tres tipos de movimientos u operaciones diferentes con las cuales se producen tres estados del mundo distintos $(2.1,2.2,2.3)$, en su proceso de toma de decisión elige ejecutar el movimiento $(2,1)$ que genera un estado al cual se le puede aplicar una única acción legal (3.1), al estado así resultante, le aplica un nuevo operador que produce el estado de conocimiento (4.1), finalmente, la persona llega al estado final encontrando la solución, una vez, ejecuta la última operación sobre el estado (4.1), 
transformándolo y produciendo con ello la respuesta al interrogante que plantea el problema. Los otros círculos en blanco $(2.2,2.3,3.2,3.3,3.4,3.5,4.1$, y 4.3$)$ representan estados sin salida, que si bien el solucionador tomó como posibles vías o caminos de exploración, en últimas, terminó descartando y abandonando la búsqueda en ellos. Conviene señalar que la gráfica en su totalidad representa el espacio del problema que una persona ha construido, entendiéndose por espacio el conjunto de todos los estados y operadores de los cuales la persona toma conciencia o logra percibir.

El investigador apoyándose en la gráfica, focalizará el posterior análisis en la secuencia de acciones que la persona realizó y los correspondientes estados por los que la misma transitó, con el propósito de identificar un patrón o pauta bajo el cual se encuentre organizada la secuencia de movimientos, es decir; la estrategia heurística utilizada por la persona. Para tal fin, el investigador usará como recursos de apoyo y complemento para el análisis, la "hoja de registros de manifestaciones conductuales" la cual le permitirá enriquecer el contenido y densidad de los datos

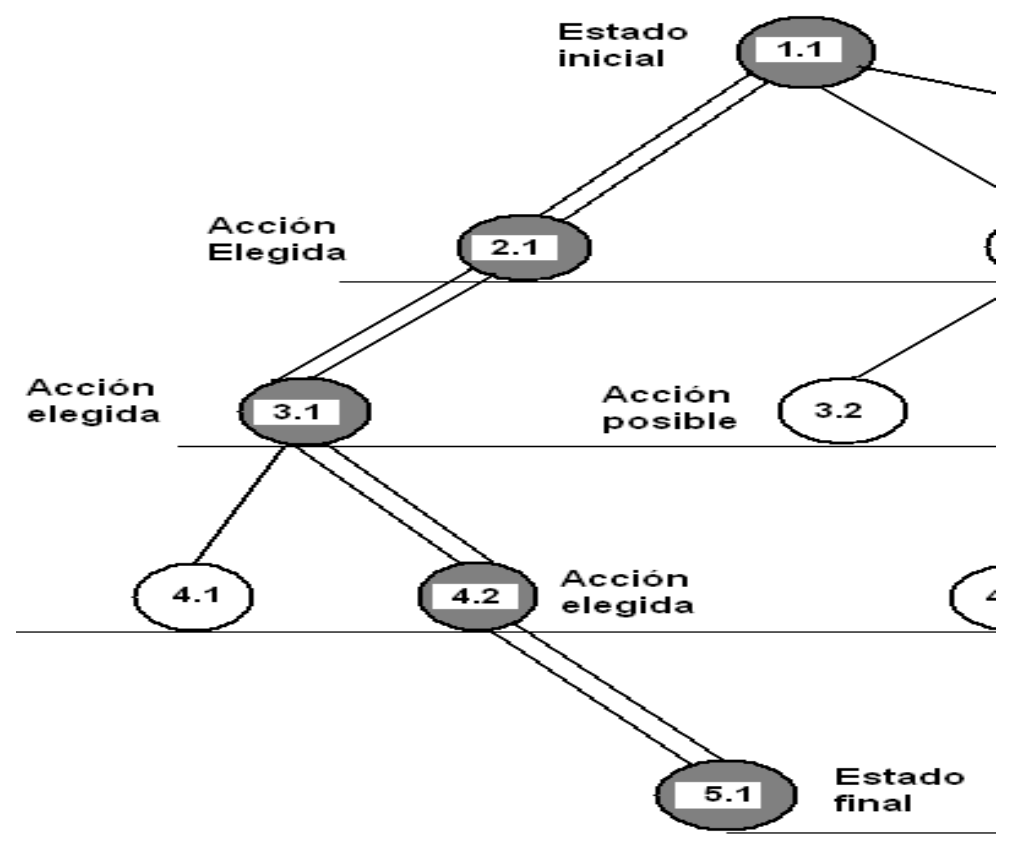

Si se parte del supuesto de que los movimientos ejecutados por el informante no difieren de manera significativa con lo que él piensa, entonces, indudablemente ha de presumirse también que el estudio de la secuencia de acciones acarrea igualmente, o al 
menos hace viable, la investigación de los procesos de razonamiento lógico y comprensión realizados por la misma persona.

\section{Sistemas de producción}

Un método alterno de análisis de los procesos de pensamiento llevados a cabo por una persona mientras resuelve un problema, consiste en codificar la estrategia utilizada por ella, en términos de producciones. Tomando en cuenta las aportaciones de la teoría psicológica. Newell \& Simon (1972) desarrollaron un sistema de reglas procedimentales dirigidas a describir la estrategia de solución implementada por el informante cuando éste resuelve un problema.

Arguyeron que las estrategias heurísticas podían ser cifradas en un lenguaje formal de programación, construido a partir de un conjunto jerárquico de reglas procedimentales, denominadas producciones; basadas en la lógica simbólica, cada una de las cuales estaría compuesta de dos partes, más específicamente, de dos variables proposicionales referidas a una condición (P) y a una acción $(\mathrm{Q})$ ambas vinculadas entre sí, a través del enlace o conectivo lógico condicionante: SI... ENTONCES..., esto es, si $(\mathrm{P}) \rightarrow(\mathrm{Q})$, que, dicho sea con las palabras empleadas: si $(\mathrm{P})$ entonces $(\mathrm{Q})$, o lo que es lo mismo; presuponiendo que se conoce el valor de verdad de la primera proposición, y se sabe a $(\mathrm{P})$ cierta y válida, entonces debe ser que $(\mathrm{Q})$ también ha de serlo.

Sin embargo, con respecto a lo dicho es conveniente hacer una pequeña digresión, únicamente a la primer proposición $(\mathrm{P})$ puede efectuársele un juicio de valor de verdad, en tanto está destinada a representar una realidad, sea ésta ficticia o material, en otras palabras, la primera proposición $(\mathrm{P})$ está referida a una condición del mundo o estado de cosas, del cual puede decirse si es falso o es verdadero.

De otro lado, no es posible atribuirle un valor de verdad a la segunda proposición $(\mathrm{Q})$, porque ella, no representa un hecho, acontecimiento o la constatación de la realidad de algo, sino que instituye más bien una acción, en consecuencia no puede ser desde este punto de vista, ni verdadera ni falsa, de esta manera la segunda proposición ocupa una dimensión práctica; no está enmarcada bajo los parámetros de descripción de una realidad sino que constituye por sí misma un acto, y en cuanto tal, no le es aplicable un valor de verdad, por cuanto no corresponde a una realidad, no es un juicio de realidad. 
De lo dicho hasta aquí se comprende que una producción posee dos componentes, uno descriptivo de una condición o estado y un componente prescriptivo que demanda la realización de una acción o movimiento, siempre y cuando, la condición (la parte sí...) de la producción se cumpla.

Usualmente los sistemas de producción suelen representarse como una lista ordenada y numerada de producciones distribuidas según el carácter prioritario que revista cada una con respecto a la otra. Las condiciones $(\mathrm{P})$ comúnmente se ubican en el extremo izquierdo y al lado de ellas, la correspondiente acción que desencadena cada una. Véase la ilustración de un posible sistema de producción diseñado para resolver la siguiente tarea.

Problema: los puntos $A, B, C$, y D forman un cuadrado, cuyo lado es $2 \mathrm{~cm}$, halle el perímetro de la región sombreada.

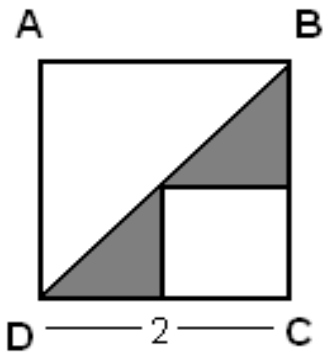

\begin{tabular}{|c|c|c|}
\hline Núm. & Condición & Acción \\
\hline & Si el perímetro está calculado. & Detener proceso. \\
\hline & Si el perímetro no está calculado. & $\begin{array}{l}\text { Establecer el objetivo de } \\
\text { calcular el perímetro. }\end{array}$ \\
\hline & $\begin{array}{l}\text { Si está establecido el objetivo de } \\
\text { hallar perímetro. }\end{array}$ & $\begin{array}{l}\text { Realizar una fórmula adecuada para } \\
\text { lograr el objetivo }\end{array}$ \\
\hline & $\begin{array}{l}\text { Si está seleccionada la formula } \\
\mathrm{P}=\mathrm{L}+\mathrm{L}+(\text { diagonal del cuadrado) }\end{array}$ & $\begin{array}{l}\text { Insertar las cantidades } \\
\text { correspondientes en ella }\end{array}$ \\
\hline & $\begin{array}{l}\text { Si falta un dato para completar el } \\
\text { cálculo del perímetro. }\end{array}$ & $\begin{array}{l}\text { Suspender objetivo de hallar } \\
\text { perímetro. }\end{array}$ \\
\hline & $\begin{array}{l}\text { Si se haya suspendido el objetivo } \\
\text { de hallar perímetro }\end{array}$ & $\begin{array}{l}\text { Establecer objetivo de hallar dato } \\
\text { faltante para calcular perímetro }\end{array}$ \\
\hline & Si está establecido el objetivo de & Realizar una fórmula adecuada para \\
\hline
\end{tabular}


Protocolo de análisis descriptivo de estrategias heurísticas aplicadas a la resolución de problemas Minotta Carlos (pp. 95-118)

\begin{tabular}{|l|l|l|l|}
\hline & hallar dato faltante & & hallar el dato faltante \\
\hline $\begin{array}{l}\text { Si está Elegida la fórmula del } \\
\text { teorema de Pitágoras } \mathrm{a}^{2}=\mathrm{b}^{2}+\mathrm{c}^{2} \\
\text { para hallar dato faltante }\end{array}$ & $\begin{array}{l}\text { Establecer objetivo de insertar las } \\
\text { cantidades conocidas en ella }\end{array}$ \\
\hline $\begin{array}{l}\text { Si se hallado el dato faltante para } \\
\text { el cálculo del perímetro }\end{array}$ & $\begin{array}{l}\text { Restablecer el objetivo de hallar } \\
\text { perímetro }\end{array}$ \\
\hline
\end{tabular}

Ilustración 1. Sistema de producción

\section{CONSIDERACIONES FINALES}

La utilidad del presente texto, radica en el desarrollo de un instrumento de descripción y análisis del proceso de resolución de problemas, que permita de develar las estrategias y tácticas empleadas por un sujeto experto o novato en un campo de las matemáticas cualquiera. Permite descubrir paso a paso, las elaboraciones mentales subyacentes a la actividad experta de algunos sujetos capaces de resolver con confianza y facilidad distintos tipos de problemas, y más importante aún, posibilita acceder a cada una de sus conjeturas, ideas creativas y captar el significado de cada línea, o gráfica realizada.

El protocolo aquí expuesto, deja de lado, la dimensión rutinaria, procedimental, y sin sentido aparente de la acción de darle solución a un ejercicio, para abrir paso al planteamiento de preguntas significativas acerca del proceso de resolución de un problema concreto. ¿Cómo lo hace, el resolutor?, ¿cuántas veces falló en su intento?, ¿por qué falló?, etc. El protocolo está diseñado para dar cuenta de las respuestas a cada una de estas preguntas, desde la voz del mismo resolutor.

\section{REFERENCIAS}

Alfaro, C. (2006). Las ideas de Polya en la resolución de problemas. las ideas de Polya en la resolución de problemas (pág. 13). Bogotá: Cuadernos de investigación y formación en educación matemática. Numero 1. Escuela de mateámicas. Universidad Nacional . 
Brito, D. (2006). La verbalización como registro para análisis en la investigación sobre lectura . Anales de Documentación, Volumen 009. Universidad de Murcia. Espinardo, España , 43-51.

Castells, L. (2007). Los protocolos de pensamientos en voz alta como instrumento para analizar el proceso de escritura. Universidad de Leida. Resla. Vol 20. pp. 27-35.

Ferreras, A. (1999). El cerebro creador. Qué hacer para que el cerebro sea mas eficaz. Madrid : Psicología Alianza Editorial, S. A.

Frías, J. (2005). Pensando en voz alta: el análisis de los protocolos verbales como método para analizar el comportamiento de los usuarios de los sistemas de recuperación de información. Memorias: Congreso de metodología de las ciencias sociales y de la salud. Universidad de Salamanca.

Garnham, A. Oakhill, J. (1994). Manual de psicología del pensamiento: Pensar y razonar. Editorial Paidós Ibérica, S. A. Barcelona.

Llera, A. (2000). Hurísitca, hipótesis y demostración en matemáticas. México, D.F. : Centro de investigaciones interdisciplinarias en ciencias y humanidades. Universidad nacional Autónoma de México .

Luria, A. (1980). Los Procesos Cognitivos. Capítulo V: Razonamiento y resolución de problemas. Editorial Fontanella S. A. España. Barcelona

Luria, A. (1981). La resolución de problemas y sus trastornos. Editorial Fontanella, S. A. Barcelona. España. Pág. 271.

López, H. (2002). Investigación cualitativa y participativa: un enfoque históricohermeneutico y crítico social en psicología y educación ambiental. Medellín, Colombia: Editorial Universidad Pontificia Bolivariana.

Manoli, P., \& Jaume, S. (2001). La enseñanza de estrategias de resolución de problemas matemáticos en la Enseñanza secundaria obligatoria ESO. Enseñanza de las ciencias 19 (2). Universidad de Leida, 297-308.

Markiewicz, M. (2004). Algunas preguntas de investigación acerca del razonamiento plausible desde la perspectiva del enfoque ontológico-semiótico. Primer congreso Internacional sobre aplicaciones y desarrollos de la teoría de las funciones semióticas en didáctica de las matemáticas. Universidad de Jaén.

Markiewicz, M. \& Etchegaray, S. (2006). algunos resultados de una investigación acerca del razonamiento plausible o congetural. Santa Rosa, La Pampa (Argentina): Universidad Nacional de Río Cuarto.

Micha, E. (2003). Matematicas discretas. México: Lumisa S.A. e C. V.

Montero, M., \& Farías, L. (2005). De la transcripción y otros aspectos artesanales de la investigación cualitativa. Internacional Journal of Qualitative Methods, 4 (1), Article 4. Retrieved , 1-14.

Newell, A., \& Simon, H. A. (1972). Human problema solving. Englewood Cliffs, NJ: Prentice-Hall. 
Pólya, G. (1989). Cómo plantear y resolver problemas. Prefacio a la primera edición en inglés. Editorial Trillas, S. A. de C. V. México D. F.

Renquena, M. (2003). el análisis de protocolo como técnica para la comprensión de los procesos de razonamiento. Universidad Pedagógica Experimental Libertador Venezuala. Laurus, Vol. 9, Núm. 16, sin mes, 79-96.

Rosen , K. (2004). Matemática discreta y sus aplicaciones (5 ed.). (J. M. Perez Morales , Trad.) España: McGraw-Hill Interamericana Editores, S.A. de C.V.

Rosen, K. Michaels, J., Gross, J. Grossman, J. \& Shier, D. (2000). Handbook of discrete and combinatorial mathematics. Nueva York: CRC Press.

Simon, H. (1972). El comportamiento administritivo. Estudio de los procesos de adopción de decisiones en la organización. Madríd (España): Agilar S.A. 